\title{
Психическое здоровье и применение антипсихотических препаратов в практике психолого-медицинского сопровождения амбулаторных больных в Азербайджане
}

\author{
Агагасан Р. Расулов ${ }^{1}$, Роя Р. Алиева ${ }^{2}$, Камаля Е. Талыбова², Айнур Г. Зейналова², \\ Гюнель Н. Алиева², Фуад Н. Исмайлов² \\ ${ }^{1}$ Азербайджанский государственный институт усовершенствования врачей имени \\ А. Алиева, г. Баку, Азербайджан \\ 2 Центр Психического Здоровья Министерства Здравоохранения Азербайджанской \\ Республики, г. Баку, Азербайджан \\ *E-mail: nauchnayastatya@yandex.ru
}

\begin{abstract}
Аннотация
Ввеление. Антипсихотические препараты имеют широкое применение в практике лечения психических расстройств как в периол острого психотического состояния, так и на стадии подАерживающей терапии. В этой связи важным требованием при назначении антипсихотиков является соответствие медикаментозного мечения принципам рационального использования лекарственных препаратов. Несмотря на актуальность этих вопросов, изучение особенностей назначений психотропных препаратов амбулаторным больным в Азербайлжане Ао настоящего времени не проводи^ось. Цель: исследование посвящено изучению применения антипсихотических препаратов в амбулаторной практике и определению фракторов, влияющих на выбор дозы препаратов и использование полифрармакотерапии.
\end{abstract}

Методы. В исследование вошли 253 пациента с различными психическими расстройствами, получающие амбулаторное лечение в Центре Психического зАоровья. Полвергавшиеся анализу переменные включали социолемографические характеристики, клинические данные, а также инорормацию о назначенном им лечении.

Результаты. Ни социолемографические, ни клинические фракторы не оказывали существенного влияния на выбор препаратов. Полавляющее большинство амбу^аторных больных получали терапию атипичными антипсихотиками, из которых наиболее часто назначался рисперилон. Аозы препаратов, как правило, были ниже рекоменАованных, и лишь у $3.2 \%$ больных отмечалось лечение относительно высокими Аозами. Более высокие Аозы лекарств ассоциировались с Аиагнозом «шизофррения» и применением полифрармакотерапии. В свою очередь, психофрармакотерапия ассоциировалась с применением традиционных нейролептиков. 
ОбсужАение результатов. Полученные результаты прелполагают, что лечение в системе амбулаторной помощи предоставляет большие возможности А^я рационального использования антипсихотических препаратов и позволяет в значительной степени персонализировать фрармакотерапию.

\section{КАючевые слова}

психические заболевания, антипсихотические препараты, полифрармакотерапия, высокие Аозы, клинические протоколы, рациональное использование, атипичные антипсихотики, нейролептики, психофрармакотерапия, амбулаторная практика

\section{Основные положения}

- при оказании психиатрической помощи амбулаторным пациентам Аозы психотропных препаратов были ниже АОз, указанных в клинических протоколах; - назначение более высокой суточной Аозы, рассчитанной в хлорпромазиновом эквиваленте, как правило, обусловлено наличием Аиагноза «шизофррения» и применением Авух и более антипсихотических препаратов;

- полавляющее большинство больных в системе амбулаторной помощи получает лечение атипичными антипсихотиками, тогАа как тралиционные нейролептики используются крайне редко;

у амбулаторных пациентов традиционные нейролептики в основном используются при проведении полиорармакотерапии.

\section{Для цитирования}

Расулов А.Р., Алиева Р.Р., Талыбова К.Е., Зейналова А. Г., Алиева Г. Н., Исмайлов Ф.Н. Психическое здоровье и применение антипсихотических препаратов в практике психолого-медицинского сопровождения амбулаторных больных в Азербайджане // Российский психологический журнал. 2018. Т. 15, № 3. С. 70-87. DOI: $10.21702 /$ rpj.2018.3.4

Материалы статьи получены 29.07.2018 


\title{
Mental Health and the Use of Antipsychotic Drugs in the Practice of Psycho-Medical Support of Outpatients in Azerbaijan
}

\author{
Agahasan R. Rasulov ${ }^{1 *}$, Roya R. Aliyeva ${ }^{2}$, Kamala E. Talibova ${ }^{2}$, Aynur G. Zeynalova ${ }^{2}$, \\ Gunel N. Aliyeva², Fuad N. Ismayilov ${ }^{2}$ \\ ${ }^{1}$ Azerbaijan State Advanced Training Institute for Doctors named after A. Aliyev, \\ Baku, Azerbaijan \\ ${ }^{2}$ National Mental Health Centre of the Ministry of Health, \\ Baku, Azerbaijan \\ *Corresponding author. E-mail: nauchnayastatya@yandex.ru
}

\begin{abstract}
Introduction. Antipsychotic drugs are widely used in treating mental disorders during acute psychotic episodes, as well as in the maintenance therapy. In this regard, adherence of drug treatment to the principles of rational use of medications is an important requirement for prescribing antipsychotics. Despite the urgency of the matter, by now no studies have addressed the issues of prescribing psychotropic drugs for outpatients in Azerbaijan. The purpose of the study is to investigate the practice of using antipsychotic drugs for outpatients and factors for the choice of the medication dose and the use of polypharmacotherapy.

Methods. In total, 253 patients with various mental disorders receiving outpatient treatment at the Mental Health Center took part in this study. The authors analyzed their sociodemographic characteristics, clinical data, and information on the prescribed treatment.
\end{abstract}

Results. Neither sociodemographic nor clinical factors had a significant influence on the choice of drugs. The vast majority of outpatients received therapy with atypical antipsychotics, where risperidone was the most frequently prescribed. The doses were generally lower than those recommended in the official guidelines; only $3.2 \%$ of patients were treated with relatively high doses. Higher doses of drugs were prescribed to the patients diagnosed with schizophrenia and associated with the use of polypharmacotherapy. In turn, psychopharmacotherapy was associated with the use of traditional neuroleptics.

Discussion. The results suggest that the treatment in the outpatient care system provides great opportunities for the rational use of antipsychotic drugs and can largely personalize pharmacotherapy. 


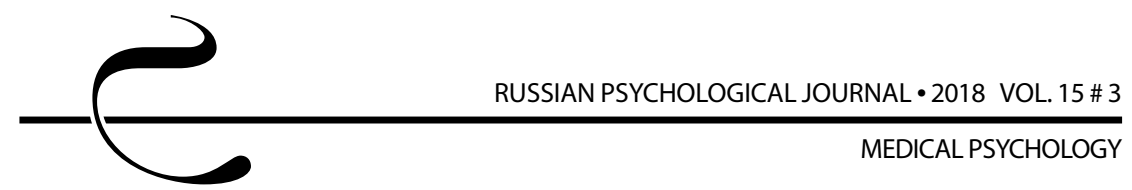

\section{Keywords}

mental diseases, antipsychotics, polypharmacotherapy, high doses, clinical protocols, rational use, atypical antipsychotics, neuroleptics, psychopharmacotherapy, outpatient practice

\section{Highlights}

- In the observed provision of psychiatric care for outpatients, the doses of psychotropic drugs were lower than the doses indicated in clinical protocols.

- A higher daily dose (in a chlorpromazine equivalent) is associated with the schizophrenia diagnosis and the use of two or more antipsychotic drugs.

- The vast majority of patients in the outpatient care system receive treatment with atypical antipsychotics, while traditional neuroleptics are rarely used.

- Outpatients receive traditional antipsychotics as a part of polypharmacotherapy.

\section{For citation}

Rasulov A. R., Aliyeva R. R., Talibova K. E., Zeynalova A.G., Aliyeva G. N., Ismayilov F. N. Mental Health and the Use of Antipsychotic Drugs in the Practice of Psycho-Medical Support of Outpatients in Azerbaijan. Rossiiskii psikhologicheskii zhurnal - Russian Psychological Journal, 2018, V. 15, no. 3, pp. 70-87 (in Russian). DOI: 10.21702/rpj.2018.3.4

Original manuscript received 29.07.2018

\section{Введение}

Реформа системы психического здоровья в Азербайджане направлена на переход от институциональной помощи к услугам, оказываемым амбулаторными учреждениями [1, 2]. Наряду с внедрением программ раннего вмешательства, работы со случаем и психосоциальной реабилитации, эффективное медикаментозное лечение, основанное на рациональном использовании психотропных препаратов, является важной частью деятельности новых учреждений психического здоровья. Рациональное использование лекарственных препаратов определяется как «получение пачиентами медикаментов в соответствии с их клиническими потребностями, в дозах отвечающих индивидуальным требованиям, в течение адекватного периода времени, а также по наименьшей стоимости для самих пациентов и для общества в целом» [3, с. 299].

Около десяти лет назад Министерством Здравоохранения были разработаны и внедрены в практику клинические протоколы по диагностике и лечению различных психических расстройств, которые носят скорее рекомендательный, нежели обязательный характер. Так же, как и во многих других странах $[4,5]$ азербайджанские психиатры сохраняют скептическое 
отношение к применению этих протоколов в своей практике. Предыдущее исследование выявило значительное несоответствие между рекомендациями, содержащимися в протоколах, и реальными назначениями лекарственной терапии больным, находящимся на стационарном лечении. В частности, было выявлено, что более половины стационарных больных лечатся традиционными нейролептиками, несмотря на рекомендуемую терапию атипичными антипсихотиками. Кроме того, более $1 \frac{1}{4}$ пациентов получали медикаменты в дозах, превышавших указанные в протоколах. В исследовании было показано, что применение высоких доз было связано с использованием полифармакотерапии, которая, вопреки действующим протоколам, часто назначалась стационарным пациентам.

В последующих обсуждениях результатов исследования со специалистами, работающими в системе стационарной помощи, было высказано мнение о том, что клинические протоколы в большей степени предназначены для ведения амбулаторных больных и не учитывают специфических особенностей медикаментозного лечения в стационарах.

Действительно, назначение лекарственной терапии в условиях стационара и в системе амбулаторной помощи имеет ряд существенных различий, которые отмечаются многими авторами $[6,7,8]$. Во-первых, пациенты, находящиеся на стационарном лечении, обнаруживают более выраженные психотические симптомы, которые являются основной причиной их госпитализации [9, 10]. Во-вторых, в большинстве случаев лечение в стационаре занимает относительно короткий промежуток времени, а амбулаторное лечение может продолжаться годами [11, 12]. В-третьих, стационарные больные, как правило, все без исключения принимают назначенные медикаменты, тогда как в амбулаторной практике большое количество пациентов самостоятельно принимают решение прекратить лекарственную терапию или принимают антипсихотики время от времени $[13,14]$. Наконец, в связи с особенностями организации психиатрической помощи в нашей стране, выбор медикаментозной терапии в стационаре ограничен государственным обеспечением лекарственными препаратами. Больные же, получающие амбулаторную помощь, не ограничены в выборе лекарств, поскольку могут самостоятельно приобретать любые назначенные им препараты в аптеках.

Целью настоящего исследования явилось изучение особенностей назначения антипсихотических препаратов больным, получающим амбулаторную помощь. В конкретные задачи исследования входило рассмотрение соответствия использования антипсихотических препаратов клиническим протоколам, включая применение атипичных антипсихотиков, назначение высоких доз и полифармакотерапии. 


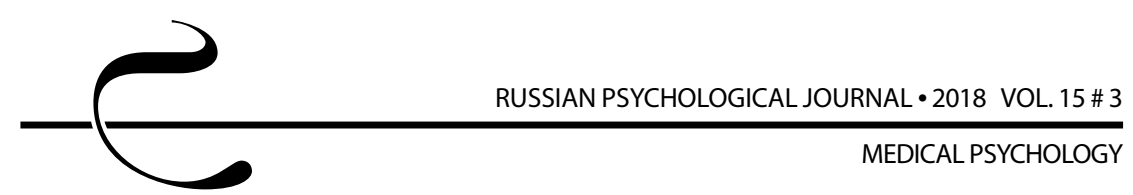

\section{Методы}

\section{Дизайн и место проведения исследования}

Настоящее исследование является кросс-секционным, натуралистическим, выполненным на конвенционной выборке пациентов, находящихся на амбулаторном лечении в Центре Психического Здоровья МЗ АР. Данное учреждение ответственно за координацию услуг в области психического здоровья на уровне страны и само оказывает внебольничную помощь взрослому населению и детям, преимущественно проживающим в г. Баку. Пациентам, обращающимся в Центр, медикаментозное лечение может быть назначено в дневном стационаре, лечебно-консультативном отделении и отделении кризисной помощи.

\section{Субъекты исследования}

Отбор пациентов осуществлялся в соответствии с рекомендациями ВОЗ для подобного рода исследований [15]. Случайным образом были изначально отобраны 600 пациентов с установленным диагнозом психического расстройства, проходивших лечение в Центре Психического Здоровья в первом полугодии 2018 г.

Из исследования были исключены пациенты младше 18 лет, лица имеющие диагноз «задержка в умственном развитии» (F7) или «психические расстройства вследствие употребления психоактивных веществ» (F2), а также больные, не принимавшие антипсихотические препараты на момент проведения исследования. Таким образом, число участников исследования составило 253 человека.

Обо всех пациентах, вошедших в исследование, были собраны данные, включавшие возраст, пол, диагноз, длительность заболевания и число госпитализаций.

\section{Источники данных и типы переменных}

Данные о медикаментозном лечении включали количество антипсихотических препаратов, их наименования, режим приема и режим дозирования. Они собиралась однократно в процессе изучения медицинской документации. В качестве антипсихотических препаратов первого поколения (FGD) рассматривались лекарственные средства, используемые в лечении шизофрении и других психических расстройств, которые обозначаются как типичные, классические или традиционные нейролептики. Под препаратами второго поколения (SGD) понимались атипичные антипсихотики, обладающие равным воздействием на продуктивные психопатологические симптомы, но имеющие несколько иной механизм действия, благодаря которому они вызывают менее выраженные побочные симптомы, чем FGD. 
Расчет дозы антипсихотиков при полифармакотерапии производился на основе конвертации доз препаратов в хлорпромазиновые эквиваленты (CPZeq). Суточная доза препарата (TDD) рассчитывалась суммированием доз препарата в CPZeq, получаемым пациентом в течение 24 часов. При этом средняя суточная доза считалась 300-600 мг CPZeq, дозы, выходящие за этот интервал, определялись соответственно как низкие или высокие. Монотерапия рассматривалась в тех случаях, когда пациент получал всего один антипсихотический препарат, тогда как назначение двух и более антипсихотиков определялось как полифармакотерапия.

\section{Статистический анализ}

Статистический анализ проводился с помощью пакета статистических программ IBM SPSS для Windows, 23.0 (Armonk, NY: IBM Corp.). Для анализа категориальных переменных, относящихся к социодемографическим и клиническим данным, использовался критерий согласия Пирсона $\chi^{2} .95 \%$ доверительный интервал $(\mathrm{Cl})$ применялся в случаях нормального распределения количественных данных, относящихся к показателям частоты назначения лекарственных препаратов. Метод множественной линейной регрессии применялся для изучения влияния клинических переменных на суммарную дозу антипсихотических препаратов. Бинарная логистическая регрессия использовалась для изучения ассоциации между характеристиками больных и психофармакотерапией. Для перевода дихотомических переменных в ординальные использовались т. н. «фиктивные» значения («0»- нет, «1»-да). Статистическая значимость для всех тестов устанавливалась на уровне $\mathrm{p}<0.05$.

\section{Результаты}

Среди пациентов, отобранных для исследования, преобладали лица женского пола ( $\chi^{2}=6.01 ; \mathrm{df}=1 ; \mathrm{p}=0.014$ ) (таблица 1). Большинство больных относились к среднему возрасту (M = 36.7; $95 \% \mathrm{Cl}$ [35.03; 38.35]), который встречался чаще других возрастных групп $\left(\chi^{2}=71.38 ; \mathrm{df}=3 ; \mathrm{p}<0.001\right)$.

Говоря о диагнозах больных, обращающихся за помощью, следует отметить наибольшую частоту диагноза шизофрении $\left(\chi^{2}=63.54 ; \mathrm{df}=4 ; \mathrm{p}<0.001\right)$. Второе место занимали органические расстройства, за которыми следовали диагнозы расстройств аффективного спектра и расстройств личности. При этом не было выявлено статистически значимых различий, связанных с длительностью болезни ( $\left.\chi^{2}=3.19 ; \mathrm{df}=4 ; \mathrm{p}=0.527\right)$. Также следует отметить, что подавляющее большинство больных не обращались за стационарной помощью. 
Таблица 1. Аемографрические и клинические характеристики пациентов

Table 1. Demographic and clinical characteristics of patients

\begin{tabular}{|c|c|}
\hline $\begin{array}{l}\text { Характеристики пациентов } \\
\text { Characteristics of patients }\end{array}$ & $\begin{array}{c}\text { Число пациентов } \\
\text { Number of patients } N \text { (\%) }\end{array}$ \\
\hline \multicolumn{2}{|l|}{$\begin{array}{c}\text { Пo^ } \\
\text { Gender }\end{array}$} \\
\hline $\begin{array}{l}\text { Мужской/Male } \\
\text { Женский/Female }\end{array}$ & $\begin{array}{l}107(42.3) \\
146(57.7)\end{array}$ \\
\hline \multicolumn{2}{|l|}{ Возраст } \\
\hline $\begin{array}{l}18-29 \\
30-44 \\
45-60 \\
\text { старше } 60 \text { / over } 60\end{array}$ & $\begin{array}{c}90(35.6) \\
94(37.2) \\
59(23.3) \\
10(4.0)\end{array}$ \\
\hline \multicolumn{2}{|l|}{$\begin{array}{l}\text { Anarно3 } \\
\text { Diagnosis }\end{array}$} \\
\hline $\begin{array}{l}\text { Шизофррения/Schizophrenia } \\
\text { Аффорективные расстройства / Affective } \\
\text { disorders } \\
\text { Органические расстройства / Organic } \\
\text { disorders } \\
\text { Рaсстройства ^ичности / Personality disorders } \\
\text { Прочие/Other }\end{array}$ & $\begin{array}{l}94(37.2) \\
42(16.6) \\
45(17.8) \\
16(6.3) \\
56(22.1)\end{array}$ \\
\hline \multicolumn{2}{|l|}{ Disease duration } \\
\hline $\begin{array}{l}\text { Ао } 1 \text { го } \Delta a / \leq 1 \text { year } \\
1-3 \text { го } \Delta \text { / } 1-3 \text { years } \\
3-5 \text { ^ет / 3-5 years } \\
\text { 5-10 ^ет / 5-10 years } \\
\text { свыше } 10 \wedge е т / \geq 10 \text { years }\end{array}$ & $\begin{array}{l}53(20.9) \\
57(22.5) \\
51(20.2) \\
40(15.8) \\
52(20.6)\end{array}$ \\
\hline \multicolumn{2}{|c|}{ Number of previous hospitalizations } \\
\hline $\begin{array}{l}\text { Нет/0 } \\
\text { ОАна/1 } \\
\text { Аве/2 } \\
\text { Три и больше / } \geq 3\end{array}$ & $\begin{array}{c}188(74.3) \\
48(19.0) \\
10(4) \\
7(2.7)\end{array}$ \\
\hline
\end{tabular}


Как показано на рисунке 1, наиболее часто больным назначался рисперидон, его получали 90 человек (35.6\%). Почти равное число больных принимали кветиапин и оланзапин, соответственно 55 (21.7\%) и 50 (19.8\%) человек. Еще 30 (11.9\%) пациентов имели в назначениях арипипразол. Другие антипсихотические препараты получали 28 (11\%) пациентов, из них 16 (6.3\%) человек принимали FGD.

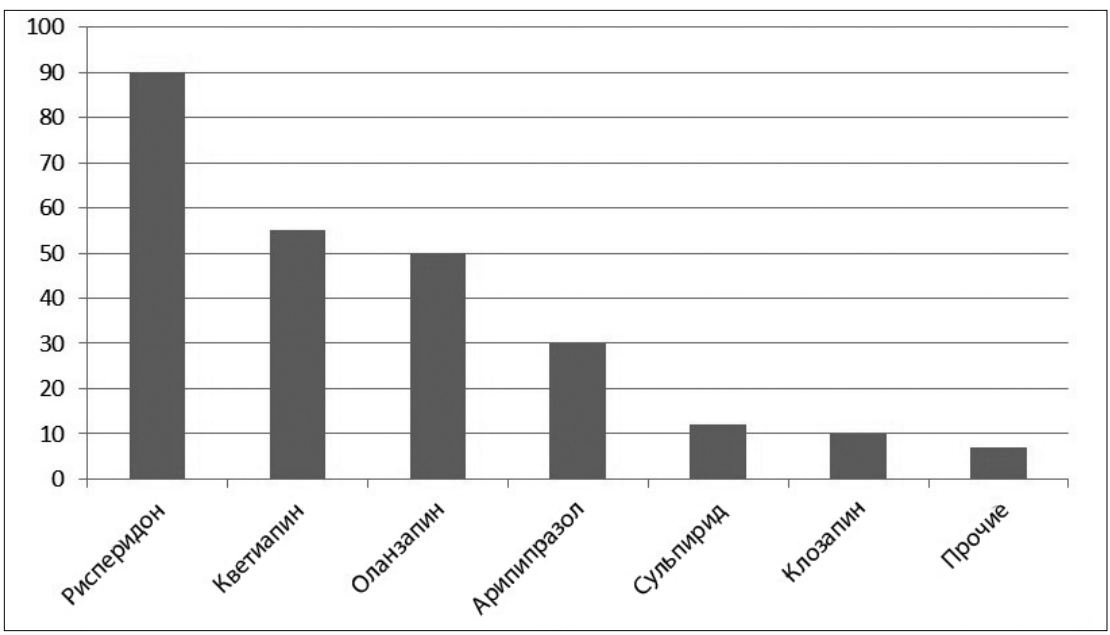

Рисунок 1. Частота назначения антипсихотических препаратов

Figure 1. Frequency of prescribing antipsychotic drugs

При конвертации на CPZeq было установлено, что антипсихотические препараты назначались в относительно низких терапевтических дозах (M = 209.4; $95 \%$ Cl [191.7; 221.1]). Лишь у 8 человек (3.2\%) CPZеq превышал 600 мг/сут.

Изучение зависимости дозы антипсихотических препаратов с социодемографическими и клиническими характеристиками больных не выявило статистически значимой связи по таким показателям, как возраст, пол, длительность заболевания и число предшествующих госпитализаций. Однако диагноз и число антипсихотиков являлись статистически значимыми предикторами суммарной суточной дозы антипсихотических препаратов. В таблице 2 показано, что предсказываемая суточная доза в CPZеq равнялась -15.925 + 94.021 (диагноз) + 187.47 (число препаратов), где диагноз кодировался как 1 - шизофрения, 0 - иной диагноз, а число препаратов определялось как общее количество антипсихотиков, которые пациент принимал на момент проведения исследования. Таким образом, больные шизофренией получали на 94 мг CPZeq 
больше, чем пациенты с другими диагнозами, а доза препарата увеличивалась на 187.47 мг в CPZеq с каждым добавленным антипсихотиком.

Таблица 2. Связь АОзы С Аиагнозом и количеством антипсихотиков

Table 2. Associations among doses, diagnosis, and number of antipsychotics

\begin{tabular}{|c|c|c|c|c|c|c|c|}
\hline $\begin{array}{c}\text { Независимая } \\
\text { переменная } \\
\text { Independent } \\
\text { variable }\end{array}$ & B & SE & $\beta$ & $t$ & $p$ & \multicolumn{2}{|c|}{$95 \% \mathrm{Cl}$} \\
\hline $\begin{array}{l}\text { Константа } \\
\text { Constant }\end{array}$ & -15.925 & 42.173 & & -0.38 & 0.706 & -98.984 & 67.135 \\
\hline $\begin{array}{l}\text { Аиагноз } \\
\text { «шизофрения» } \\
\text { Diagnosis } \\
\text { Schizophrenia }\end{array}$ & 94.021 & 16.999 & 0.318 & 5.53 & 0.000 & 5.53 & 127.5 \\
\hline $\begin{array}{l}\text { Число } \\
\text { препаратов } \\
\text { Antipsychotics } \\
\text { (n) }\end{array}$ & 187.47 & 41.447 & 0.26 & 4.52 & 0.000 & 105.84 & 269.1 \\
\hline $\begin{array}{l}R^{2}=0.200 ; N=25 \\
p<0.001\end{array}$ & $F(d f=2$; & $=252)=$ & .272 & & & & \\
\hline
\end{tabular}

Анализ методом бинарной логистической регрессии для установления связи полифармакотерапии с социодемографическими и клиническими факторами не выявил существенного влияния возраста и пола больных, а также длительности их заболевания и числа предшествующих госпитализаций на лечение двумя и более препаратами. В то же время результаты анализа указывают на наличие статистически значимой ассоциации между диагнозом шизофрения и лечением FGA и полифармакотерапией $\left(\chi^{2}=9.433\right.$; $\mathrm{p}=0.009)$. Как видно из таблицы 3, лечение FGA в 17 раз, а наличие диагноза шизофрения почти в 8 раз повышает шансы применения одновременно нескольких антипсихотических препаратов. 
Таблица 3. Связь к^инических характеристик с полифрармакотерапией

Table 3. Relationship between clinical characteristics and polypharmacotherapy

\begin{tabular}{|c|c|c|c|c|c|}
\hline $\begin{array}{l}\text { Показатель } \\
\text { Characteristic }\end{array}$ & $\beta$ & Wald & $p$ & OR & $95 \% \mathrm{Cl}$ \\
\hline $\begin{array}{l}\text { Лечение FGA } \\
\text { FGA treatment }\end{array}$ & 2.829 & 7.274 & 0.007 & 16.931 & $\begin{array}{c}2.167 ; \\
132.311\end{array}$ \\
\hline $\begin{array}{l}\text { Аиагноз } \\
\text { «шизофрения» }\end{array}$ & & & & & \\
\hline $\begin{array}{l}\text { Diagnosis } \\
\text { Schizophrenia }\end{array}$ & 2.046 & 4.396 & 0.036 & 7.736 & $1.143 ; 52.378$ \\
\hline
\end{tabular}

\section{Обсуждение результатов}

Настоящее исследование выполнено среди пациентов, получающих амбулаторное лечение, и оно является продолжением предыдущего исследования лекарственных назначений у стационарных больных. Сравнение результатов обоих исследований выявило существенные различия в использовании антипсихотических препаратов в зависимости от типа оказываемой помощи. В частности, в стационарах больше половины больных продолжают лечиться традиционными нейролептиками, тогда как в системе амбулаторной помощи применяются преимущественно атипичные антипсихотические препараты. Указанные различия могут объяснять данные ряда исследований о том, что атипичные антипсихотики чаще назначаются при менее тяжелых расстройствах, тогда как в более острых состояниях врачи склонны больше полагаться на классические нейролептики $[16,17,18]$. Кроме того, различия между стационарной и амбулаторной практикой могут быть обусловлены стремлением сократить расходы на лечение в государственных больницах, где предпочтение отдается более дешевым антипсихотикам первого поколения. Одновременно, малозатратная амбулаторная помощь в большей степени ориентирована на эффективность, снижение риска развития экстрапирамидных симптомов и комплаентность к лечению. В этом отношении атипичные антипсихотические препараты обладают преимуществом перед традиционными нейролептиками [19, 20, 21].

Так же, как и среди стационарных больных, наиболее часто используемым у амбулаторных пациентов атипичным антипсихотиком был рисперидон. Вместе с тем в системе амбулаторной помощи гораздо чаще назначались 


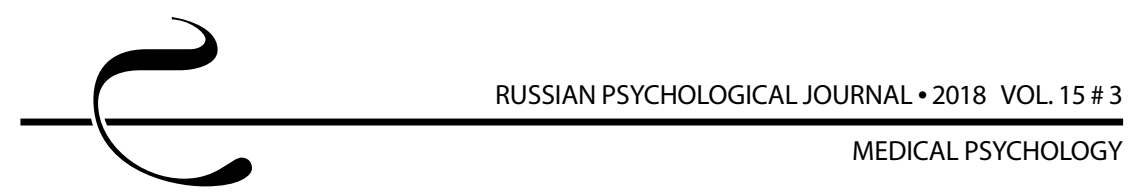

и другие антипсихотические препараты. Разнообразие назначаемых антипсихотиков в амбулаторной практике может быть обусловлено более благоприятными условиями для их использования и стремлением врачей персонализировать лечение [22, 23].

Говоря о режиме дозирования, следует отметить, что амбулаторные пациенты получали антипсихотики в относительно небольших дозах - меньших, чем рекомендовано в клинических протоколах. По мнению некоторых авторов, это обстоятельство связано с тем, что в клинических испытаниях, на которых основываются действующие протоколы, назначаются дозы, рекомендуемые компаниями-разработчиками лекарственных препаратов, тогда как обычное амбулаторное лечение позволяет обойтись более низкими дозами антипсихотиков $[24,25]$. Как показывает настоящее исследование, более высокие дозы антипсихотиков ассоциируются с диагнозом шизофрении, тогда как многие пациенты, находящиеся на амбулаторном лечении, имели другие диагнозы, поддающиеся лечению низкими дозами антипсихотиков. Следует также отметить, что большинство больных шизофренией на момент проведения исследования, находясь в ремиссии, получали поддерживающую терапию меньшими дозами препаратов, которые ассоциируются с лучшей переносимостью лечения и функциональной адаптацией больных [26, 27, 28].

Как и следовало ожидать, высокие дозы антипсихотических препаратов ассоциировались с применением полифармакотерапии. В свою очередь, применение полифармакотерапии связано с диагнозом шизофрении и лечением традиционными нейролептиками. К аналогичным выводам приходят и другие авторы [29, 30]. В отличие от исследования, выполненного среди стационарных больных, в данном исследовании не нашла подтверждения зависимость выбора препарата от социодемографических характеристик больных или длительности их заболевания. В этом аспекте можно констатировать, что лечение антипсихотическими препаратами в системе амбулаторной помощи в большей степени соответствует принятым в стране клиническим протоколам.

В данном исследовании использован кросс-секционный дизайн, который накладывает определенные ограничения на интерпретацию причинно-следственных связей между изучаемыми переменными. Важные факторы, такие как степень тяжести состояния пациентов, выраженность побочных явлений, наличие терапевтической резистентности, потенциально влияющие на режим дозирования и психофармакотерапию, не учитывались в настоящем исследовании. Исследование ограничивалось только амбулаторными больными из расположенного в столице Центра Психического Здоровья, поэтому его результаты не вполне применимы к амбулаторным пациентам, получающим помощь в других учреждениях и регионах страны. 


\section{Литература}

1. Ismayilov F. Mental health services in Azerbaijan // Bulletin of the Board of International Affairs of the Royal College of Psychiatrists. 2004. Issue 3. P. 16-17. URL: https://www.researchgate.net/publication/322374144_Mental_health_ services in Azerbaijan/fulltext/5a56b6ec0f7e9bf2a536cec5/3223741 44 Mental health services in Azerbaijan.pdf?origin=publication detail (Accessed 21.08.2018).

2. Расулов А. Р. Развитие системы охраны психического здоровья в Азербайджане // Казанский медицинский журнал. 2017. Т. 98, № 4. С. 656-661.

3. Rational use of Drugs: Report of the Conference of Experts, Nairobi, 25-29 November 1985. Geneva: World Health Organization, 1987. 329 p.

4. Fleischhacker W. W., Uchida H. Critical review of antipsychotic polypharmacy in the treatment of schizophrenia // International Journal of Neuropsychopharmacology. 2014. Vol. 17, Issue 7. P. 1083-1093. DOI: 10.1017/S1461145712000399

5. Saddichha S., Chaturvedi S. K. Clinical Practice Guidelines in Psychiatry: More Confusion Than Clarity? A Critical Review and Recommendation of a Unified Guideline //ISRN Psychiatry. 2014. Article ID 828917.DOI: $10.1155 / 2014 / 828917$

6. Paton C., Barnes T. R. E., Cavanagh M.-R, Taylor D., Lelliott P. High-dose and combination antipsychotic prescribing in acute adult wards in the UK: The challenges posed by p.r.n. prescribing // The British Journal of Psychiatry. 2008. Vol. 192, Issue 6. P. 435-439. DOI: 10.1192/bjp.bp.107.042895

7. Romstadt N., Wonson E. Outcomes comparison of long-acting injectable antipsychotic initiation in treatment-naïve veterans in the inpatient versus outpatient setting //The Mental Health Clinician. 2018. Vol. 8, № 1. P. 24-27. DOI: $10.9740 / \mathrm{mhc} .2018 .01 .024$

8. EdlingerM., RettenbacherM. A., Kemmler G., Biedermann F., Widschwendter C. G., Fleischhacker W. W., Hofer A. Prescribing Practice in Inpatients Versus Outpatients With Schizophrenia Initiating Treatment With Second-Generation Antipsychotics: A Naturalistic Follow-Up Study // Journal of Clinical Psychopharmacology. 2016. Vol. 36, Issue 6. P. 621-627. DOI: $10.1097 /$ JCP.0000000000000573

9. Barbui C., Biancosino B., Esposito E., et al. Factors associated with antipsychotic dosing in psychiatric inpatients: a prospective study // International Clinical Psychopharmacology. 2007. Vol. 22, Issue 4. P. 221-225. DOI: $10.1097 /$ YIC.0b013e3281084ea8

10. Harder D. W., Strauss J. S., Greenwald D. F., Kokes R. F., Ritzler B. A., Gift T. E. Life events and psychopathology severity: comparisons between psychiatric outpatients and inpatients // Journal of Clinical Psychology. 1989. Vol. 45. P. 202-209. 
11. Warnke I., Rössler W., Herwig U. Does psychopathology at admission predict the length of inpatient stay in psychiatry? Implications for financing psychiatric services // BMC Psychiatry. 2011. Vol. 11. P. 120.

12. Tiihonen J., Walhbeck K., Lönnqvist J., et al. Effectiveness of antipsychotic treatments in a nationwide cohort of patients in community care after first hospitalisation due to schizophrenia and schizoaffective disorder: observational follow-up study // BMJ.2006.Vol.333. P. 224. DOI:10.1136/bmj.38881.382755.2F

13. Harrow M., Jobe T. H., Faull R. N. Do all schizophrenia patients need antipsychotic treatment continuously throughout their lifetime? A 20-year longitudinal study // Psychological Medicine. 2012. Vol. 42, Issue 10. P. 2145-2155. DOI: $10.1017 /$ S0033291712000220

14. Offord S., Lin J., Wong B., Mirski D., Baker R. A. Impact of Oral Antipsychotic Medication Adherence on Healthcare Resource Utilization Among Schizophrenia Patients with Medicare Coverage // Community Mental Health Journal. 2013. Vol. 49, Issue 6. P. 625-629. DOI: 10.1007/s10597-013-9638-y

15. How to Investigate Drug Use in Health Facilities: Selected Drug Indicators. Geneva: World Health Organization, 1993. 92 p.

16. Gaebel W., Riesbeck M., Janssen B., Schneider F., Held T., Mecklenburg H., Sass $H$. Atypical and typical neuroleptics in acute schizophrenia and related delusional disorders. Drug choice, switching and outcome under naturalistic treatment conditions // European Archives of Psychiatry and Clinical Neuroscience. 2003. Vol. 253. P. 175-184.

17. Bernardo M., Coma A., Ibáñez C., Zara C., Bari J. M., Serrano-Blanco A. Antipsychotic polypharmacy in a regional health service: a population-based study // BMC Psychiatry. 2012. Vol. 12. P. 42. DOI: 10.1186/1471-244X-12-42

18. Satterthwaite T. D., Wolf D. H., Rosenheck R. A., Gur R. E., Caroff S. N. A MetaAnalysis of the Risk of Acute Extrapyramidal Symptoms With Intramuscular Antipsychotics for the Treatment of Agitation // The Journal of Clinical Psychiatry. 2008. Vol. 69 (12). P. 1869-1879.

19. Zhang J.-P., Gallego J. A., Robinson D. G., Malhotra A. K., Kane J. M., Correll C. U. Efficacy and safety of individual second-generation vs first-generation antipsychotics in first-episode psychosis: a systematic review and metaanalysis // International Journal of Neuropsychopharmacology / Collegium Internationale Neuropsychopharmacologicum. 2013. Vol. 16, Issue 6. P. 1205-1218. DOI: $10.1017 /$ S1461145712001277

20. Gründer G., Heinze M., Cordes J., Mühlbauer B., Juckel G., Schulz C., Rüther E., Timm J. Effects of first-generation antipsychotics versus second-generation antipsychotics on quality of life in schizophrenia: a double-blind, randomised study //The Lancet Psychiatry. 2016. Vol. 3, Issue 8. P. 717-729. DOI: 10.1016/ S2215-0366(16)00085-7 
21. Ponizovsky A. M., Marom E., Ben-Laish M., Barash I., Weizman A., Schwartzberg E. Trends in the use of antipsychotics in the Israeli inpatient population, 2004-2013 // Israel Journal of Health Policy Research. 2016. Vol. 5. P. 16. DOI: 10.1186/s13584-016-0074-7

22. Meltzer H. Y. New Trends in the Treatment of Schizophrenia // CNS \& Neurological Disorders - Drug Targets. 2017. Vol. 16, Issue 8. P. 900-906. DOI: $10.2174 / 1871527316666170728165355$

23. Li M. Antipsychotic-induced sensitization and tolerance: Behavioral characteristics, developmental impacts, and neurobiological mechanisms // Journal of Psychopharmacology. 2016. Vol. 30, Issue 8. P. 749-770.

24. Hanssens L., De Hert M., Wampers M., et al. Pharmacological treatment of ambulatory schizophrenic patients in Belgium // Clinical Practice and Epidemiology in Mental Health. 2006. Vol. 2.P. 11.DOI: 10.1186/1745-0179-2-11

25. Buckley P. F., Correll C. U. Strategies for dosing and switching antipsychotics for optimal clinical management //The Journal of Clinical Psychiatry. 2008. Vol. 69, Suppl. 1. P. 4-17.

26. Volavka J., Vevera J. Very long-term outcome of schizophrenia // The International Journal of Clinical Practice. 2018. Vol. 72, Issue 7. e13094. DOI: 10.1111/ijcp.13094

27. Moilanen J. M., Haapea M., Jääskeläinen E., Veijola J. M., Isohanni M. K., Koponen H. J., Miettunen J. Long-term antipsychotic use and its association with outcomes in schizophrenia - the Northern Finland Birth Cohort 1966 // European Psychiatry. 2016. Vol. 36. P.7-14.DOI: 10.1016/j.eurpsy.2016.03.002 28. Uchida H., Suzuki T., Takeuchi H., Arenovich T., Mamo D. C. Low Dose vs Standard Dose of Antipsychotics for Relapse Prevention in Schizophrenia: Meta-analysis // Schizophrenia Bulletin. 2011. Vol. 37, Issue 4. P. 788-799. DOI: $10.1093 /$ schbul/sbp149

29. Fisher M. D., Reilly K., Isenberg K., Villa K. F. Antipsychotic patterns of use in patients with schizophrenia: polypharmacy versus monotherapy // BMC Psychiatry. 2014. Vol. 14. P. 341. DOI: $10.1186 /$ s12888-014-0341-5

30. McMillan S. S, Jacobs S., Wilson L., et al. Antipsychotic prescribing for vulnerable populations: a clinical audit at an acute Australian mental health unit at two-time points // BMC Psychiatry. 2017. Vol. 17. P. 139. DOI: $10.1186 /$ s12888-017-1295-1

\section{References}

1. Ismayilov F. Mental health services in Azerbaijan. Bulletin of the Board of International Affairs of the Royal College of Psychiatrists, 2004, Issue 3, pp. 16-17. Available at: https://www.researchgate.net/publication/322374144 Mental health services in Azerbaijan/fulltext/5a56b6ec0f7e9bf2a536cec5/32237 


\section{Mental health services in Azerbaijan.pdf?origin=publication detail} (Accessed 21 August 2018).

2. Rasulov A. R. Development of mental health system in Azerbaijan. Kazanskii meditsinskii jurnal - Kazan Medical Journal, 2017, V. 98, no. 4, pp.656-661 (in Russian).

3. Rational use of Drugs: Report of the Conference of Experts, Nairobi, 25-29 November 1985. Geneva: World Health Organization, 1987. 329 p.

4. Fleischhacker W. W., Uchida H. Critical review of antipsychotic polypharmacy in the treatment of schizophrenia. International Journal of Neuropsychopharmacology. 2014.V. 17, Issue 7, pp. 1083-1093. DOI: $10.1017 /$ $\underline{\mathrm{S} 1461145712000399}$

5. Saddichha S., Chaturvedi S. K. Clinical practice guidelines in psychiatry: More confusion than clarity? A critical review and recommendation of a unified guideline. ISRN Psychiatry, 2014. Article ID 828917. DOI: $10.1155 / 2014 / 828917$

6. Paton C., Barnes T. R. E., Cavanagh M.-R, Taylor D., Lelliott P. High-dose and combination antipsychotic prescribing in acute adult wards in the UK: The challenges posed by p.r.n. prescribing. The British Journal of Psychiatry, 2008, V. 192, Issue 6, pp. 435-439. DOI: 10.1192/bjp.bp.107.042895

7. Romstadt N., Wonson E. Outcomes comparison of long-acting injectable antipsychotic initiation in treatment-naïve veterans in the inpatient versus outpatient setting. The Mental Health Clinician, 2018, V. 8, no. 1, pp. 24-27. DOI: $10.9740 / \mathrm{mhc} .2018 .01 .024$

8. Edlinger M., Rettenbacher M. A., Kemmler G., Biedermann F., Widschwendter C. G., Fleischhacker W.W., Hofer A. Prescribing practice in inpatients versus outpatients with schizophrenia initiating treatment with secondgeneration antipsychotics: A naturalistic follow-up study. Journal of Clinical Psychopharmacology, 2016, V. 36, Issue 6, pp. 621-627. DOI: 10.1097/ JCP.0000000000000573

9. Barbui C., Biancosino B., Esposito E., et al. Factors associated with antipsychotic dosing in psychiatric inpatients: a prospective study. International Clinical Psychopharmacology, 2007, V. 22, Issue 4, pp. 221-225. DOI: $10.1097 /$ YIC.0b013e3281084ea8

10. Harder D. W., Strauss J. S., Greenwald D. F., Kokes R. F., Ritzler B. A., Gift T. E. Life events and psychopathology severity: comparisons between psychiatric outpatients and inpatients. Journal of Clinical Psychology, 1989, V. 45, pp. 202-209.

11. Warnke I., Rössler W., Herwig U. Does psychopathology at admission predict the length of inpatient stay in psychiatry? Implications for financing psychiatric services. BMC Psychiatry, 2011, V. 11, p. 120. 
12. Tiihonen J., Walhbeck K., Lönnqvist J., et al. Effectiveness of antipsychotic treatments in a nationwide cohort of patients in community care after first hospitalisation due to schizophrenia and schizoaffective disorder: observational follow-up study. BMJ, 2006, V. 333, p. 224. DOI: $10.1136 /$ bmj.38881.382755.2F

13. Harrow M., Jobe T. H., Faull R. N. Do all schizophrenia patients need antipsychotic treatment continuously throughout their lifetime? A 20-year longitudinal study. Psychological Medicine, 2012, V. 42, Issue 10, pp. 2145-2155. DOI: $10.1017 /$ S0033291712000220

14. Offord S., Lin J., Wong B., Mirski D., Baker R. A. Impact of oral antipsychotic medication adherence on healthcare resource utilization among schizophrenia patients with Medicare coverage. Community Mental Health Journal, 2013, V. 49, Issue 6, pp. 625-629. DOI: 10.1007/s10597-013-9638-y

15. How to investigate drug use in health facilities: Selected drug indicators. Geneva: World Health Organization, 1993. 92 p.

16. Gaebel W., Riesbeck M., Janssen B., Schneider F., Held T., Mecklenburg H., Sass $\mathrm{H}$. Atypical and typical neuroleptics in acute schizophrenia and related delusional disorders. Drug choice, switching and outcome under naturalistic treatment conditions. European Archives of Psychiatry and Clinical Neuroscience, 2003, V. 253, pp. 175-184.

17. Bernardo M., Coma A., Ibáñez C., Zara C., Bari J. M., Serrano-Blanco A. Antipsychotic polypharmacy in a regional health service: a population-based study. BMC Psychiatry, 2012, V. 12, p. 42. DOI: $10.1186 / 1471-244 \mathrm{X}-12-42$

18. Satterthwaite T. D., Wolf D. H., Rosenheck R. A., Gur R. E., Caroff S. N. A metaanalysis of the risk of acute extrapyramidal symptoms with intramuscular antipsychotics for the treatment of agitation. The Journal of Clinical Psychiatry, 2008, V. 69 (12), pp. 1869-1879.

19. Zhang J.-P., Gallego J. A., Robinson D. G., Malhotra A. K., Kane J. M., Correll C. U. Efficacy and safety of individual second-generation vs firstgeneration antipsychotics in first-episode psychosis: a systematic review and meta-analysis. International Journal of Neuropsychopharmacology, Collegium Internationale Neuropsychopharmacologicum, 2013, V. 16, Issue 6, pp. 1205-1218. DOI: $10.1017 /$ S1461145712001277

20. Gründer G., Heinze M., Cordes J., Mühlbauer B., Juckel G., Schulz C., Rüther E., Timm J. Effects of first-generation antipsychotics versus second-generation antipsychotics on quality of life in schizophrenia: a double-blind, randomised study. The Lancet Psychiatry, 2016, V. 3, Issue 8, pp. 717-729. DOI: 10.1016/ S2215-0366(16)00085-7

21. Ponizovsky A. M., Marom E., Ben-Laish M., Barash I., Weizman A., Schwartzberg E. Trends in the use of antipsychotics in the Israeli inpatient 
population, 2004-2013. Israel Journal of Health Policy Research, 2016, V. 5, p. 16. DOI: 10.1186/s13584-016-0074-7

22. Meltzer H. Y. New trends in the treatment of schizophrenia. CNS \& Neurological Disorders - Drug Targets, 2017, V. 16, Issue 8, pp. 900-906. DOI: $10.2174 / 1871527316666170728165355$

23. Li M. Antipsychotic-induced sensitization and tolerance: Behavioral characteristics, developmental impacts, and neurobiological mechanisms. Journal of Psychopharmacology, 2016, V. 30, Issue 8, pp. 749-770.

24. Hanssens L., De Hert M., Wampers M., et al. Pharmacological treatment of ambulatory schizophrenic patients in Belgium. Clinical Practice and Epidemiology in Mental Health, 2006, V. 2, p. 11. DOI: 10.1186/1745-0179-2-11

25. Buckley P. F., Correll C. U. Strategies for dosing and switching antipsychotics for optimal clinical management. The Journal of Clinical Psychiatry, 2008, V. 69, Suppl. 1, pp. 4-17.

26. Volavka J., Vevera J.Very long-term outcome of schizophrenia. The International Journal of Clinical Practice, 2018, V. 72, Issue 7, e13094. DOI: 10.1111/ijcp.13094

27. Moilanen J. M., Haapea M., Jääskeläinen E., Veijola J. M., Isohanni M. K., Koponen $\mathrm{H}$. J., Miettunen J. Long-term antipsychotic use and its association with outcomes in schizophrenia - the Northern Finland Birth Cohort 1966. European Psychiatry, 2016, V. 36, pp. 7-14. DOI:10.1016/j.eurpsy.2016.03.002

28. Uchida H., Suzuki T., Takeuchi H., Arenovich T., Mamo D. C. Low dose vs standard dose of antipsychotics for relapse prevention in schizophrenia: Meta-analysis. Schizophrenia Bulletin, 2011, V. 37, Issue 4, pp. 788-799. DOI: $10.1093 /$ schbul/sbp149

29. Fisher M. D., Reilly K., Isenberg K., Villa K. F. Antipsychotic patterns of use in patients with schizophrenia: polypharmacy versus monotherapy. BMC Psychiatry, 2014, V. 14, p. 341. DOI: 10.1186/s12888-014-0341-5

30. McMillan S. S, Jacobs S., Wilson L., et al. Antipsychotic prescribing for vulnerable populations: a clinical audit at an acute Australian mental health unit at two-time points. BMC Psychiatry, 2017, V. 17, p. 139. DOI: $10.1186 /$ $\underline{\text { s12888-017-1295-1 }}$ 